\title{
Genes involved in mitochondrial biogenesis/function are induced in response to bilio-pancreatic diversion in morbidly obese individuals with normal glucose tolerance but not in type 2 diabetic patients
}

\author{
M. I. Hernández-Alvarez • C. Chiellini • M. Manco • \\ D. Naon • M. Liesa • M. Palacín • G. Mingrone • \\ A. Zorzano
}

Received: 13 January 2009 / Accepted: 20 April 2009 /Published online: 6 June 2009

(C) Springer-Verlag 2009

\begin{abstract}
Aims/hypothesis The mechanisms allowing normalisation of insulin sensitivity and reversal of type 2 diabetes after bilio-pancreatic diversion (BPD) have not been elucidated. We studied whether the expression of genes relevant to mitochondrial biogenesis/function is induced in response to BPD and whether the response differs between morbidly obese patients with normal glucose tolerance (NGT) and patients with type 2 diabetes.

Methods The effect of stable weight reduction after BPD on metabolic variables and expression of nuclear genes encoding for mitochondrial proteins or regulators of mitochondrial function was investigated in skeletal muscle. Insulin sensitivity was assessed by euglycaemic-hyperinsulinaemic clamp and substrate oxidation by indirect calorimetry.
\end{abstract}

G. Mingrone and A. Zorzano share senior authorship.

Electronic supplementary material The online version of this article (doi:10.1007/s00125-009-1403-y) contains supplementary material, which is available to authorised users.

M. I. Hernández-Alvarez $\cdot$ D. Naon $\cdot$ M. Liesa $\cdot$ M. Palacín •

A. Zorzano $(\triangle)$

Institute for Research in Biomedicine (IRB Barcelona),

Parc Cientific de Barcelona, c/ Baldiri Reixac 10-12,

08028 Barcelona, Spain

e-mail: antonio.zorzano@irbbarcelona.org

M. I. Hernández-Alvarez • D. Naon • M. Liesa • M. Palacín •

A. Zorzano

Departament de Bioquímica i Biologia Molecular,

Facultat de Biologia, Universitat de Barcelona,

Barcelona, Spain
Results Both NGT and type 2 diabetic patients showed a net improvement of insulin sensitivity, with the latter also showing blood glucose normalisation. NGT patients had a large increase in glucose oxidation and substantial reduction in lipid oxidation. In contrast, type 2 diabetic patients had a blunted response to BPD in terms of glucose oxidation. NGT patients showed increased expression of genes encoding mitofusin 2 , porin or citrate synthase; no significant changes were detected in diabetic patients. The expression of genes regulating mitochondrial activity (PGC-1 $\beta$ [also known as PPARGC1B], PGC-1 $\alpha$ [also known as PPARGC1A], PPAR [also known as PPARD], SIRT1) was induced only in NGT patients.

Conclusions/interpretation These findings indicate that weight loss after BPD exerts a beneficial effect on insulin sensitivity via mechanisms that are independent of the

M. I. Hernández-Alvarez • D. Naon • M. Liesa · A. Zorzano CIBER on Diabetes and Associated Metabolic Diseases (CIBERDEM)

URL: www.ciberdem.org/

C. Chiellini · G. Mingrone

Institute of Internal Medicine, School of Medicine, Catholic University,

Rome, Italy

M. Manco

Research Institute, Bambino Gesù Hospital,

Rome, Italy 
expression of genes involved in mitochondrial biogenesis/ activity. Furthermore, the observation that gene expression is not altered with weight loss in type 2 diabetic patients while it is induced in NGT patients suggests a heritable component.

Keywords Bariatric surgery - Mitochondria - Mitofusin 2 . Obesity $\cdot$ PGC- $1 \alpha \cdot$ PGC-1 $\beta \cdot$ PPAR $\delta \cdot$ SIRT $\cdot$ Skeletal muscle $\cdot$ Type 2 diabetes

$\begin{array}{ll}\text { Abbreviations } \\ \text { ANCOVA } & \text { Analysis of covariance } \\ \text { BPD } & \text { Bilio-pancreatic diversion } \\ \text { FFM } & \text { Free-fat mass } \\ \text { MFN2 } & \text { Mitofusin } 2 \\ \text { NGT } & \text { Normal glucose tolerance } \\ \text { PGC-1 } & \text { Peroxisome proliferator-activated receptor } \gamma \\ & \text { coactivator 1 } \\ \text { PPAR- } \delta & \text { Peroxisome proliferator-activated receptor- } \delta \\ \text { QUICKI } & \text { Quantitative insulin sensitivity check index } \\ \text { REE } & \text { Resting energy expenditure } \\ \text { RQ } & \text { Respiratory quotient } \\ \text { SIRT1 } & \text { Silent mating type information regulation } 2 \\ & \text { homologue 1 }\end{array}$

\section{Introduction}

The role of mitochondrial metabolism in the pathophysiology of insulin resistance is currently much debated, particularly with regard to diabetic individuals. On the one hand some scientists have proposed that compromised mitochondrial oxidative function, particularly in skeletal muscle, is responsible for lipid accumulation and development of insulin resistance $[1,2]$. There is thus no doubt that insulin-resistant, obese individuals affected by type 2 diabetes have approximately $30 \%$ less mitochondria in their skeletal muscles than age-matched healthy controls [3]. Some studies have reported that the mitochondrial alterations found in muscle of type 2 diabetic patients reflect a functional impairment of mitochondria, since these alterations are present even after correction by mitochondrial mass [4, 5]. However, in other studies, differences in electron transport chain or in oxygen consumption have not been detected after correction by surrogates of mitochondrial mass $[1,6]$. On the other hand, other scientists have serious reservations about a causative role of mitochondrial dysfunction in this condition. Thus, Asian Indians displaying higher mtDNA content and increased oxidative enzyme activity are more insulin resistant than age-, sex- and BMImatched North American counterparts [7]. Furthermore, no deficit in mitochondrial function was found in skeletal muscles from obese patients in early or advanced stages of type 2 diabetes [8].

Weight reduction and physical exercise are the best approaches to improve insulin sensitivity [9]. However, compliance to lifestyle changes has been proven to be poor in the long term [10]. In the last decades, bariatric surgery has emerged as a potential therapy for diabetes [11]. Biliopancreatic diversion (BPD) is a bariatric surgical technique characterised by massive weight loss mainly due to lipid malabsorption $[12,13]$. BPD causes a net improvement in insulin sensitivity, long before normalisation of body weight [14]. In addition, BPD regulates substrate oxidation, modulating the expression of genes involved in lipid synthesis $[15,16]$ and oxidation $[15,17]$ in both muscle and adipose tissue.

BPD operation caused a net improvement of insulin sensitivity in type 2 diabetic and in non-diabetic patients [14]. We have previously described increased MFN2 mRNA expression in skeletal muscle from morbidly obese patients with normal glucose tolerance (NGT) after BPD [18]. In the present study, we aimed to determine whether the expression of genes involved in mitochondrial biogenesis/function was induced in response to BPD. To this end we selected: (1) nuclear genes that regulate mitochondrial biogenesis such as $P G C$ - $1 \alpha$ (also known as PPARGC1A), $P G C-1 \beta$ (also known as PPARGC1B) and PPAR $\delta$ (also known as $P P A R D)$; (2) genes that regulate mitochondrial metabolism and fusion such as $\operatorname{MFN2}$ [19, 20]; (3) genes that regulate peroxisome proliferator-activated receptor $\gamma$ coactivator $1(P G C-1 \alpha)$ such as SIRT1 [21, 22]; and (4) genes that encode for constitutive proteins such as porin or citrate synthase.

In addition, and being interested in the mechanisms leading to the reversal of diabetes after BPD, we investigated the effect of comparable weight loss caused by BPD on the expression of those genes mentioned above, and whether the response differed between morbid obese NGT or type 2 diabetic patients. All patients were characterised before and after weight loss with respect to insulin sensitivity by the euglycaemic-hyperinsulinaemic clamp; their glucose and lipid oxidation was assessed by the respiratory chamber.

\section{Methods}

Patients We studied 21 morbidly obese patients undergoing BPD. Before surgery, 11 patients had NGT and ten had overt type 2 diabetes. Diabetes was newly diagnosed in all diabetic patients at the time of the study, and they were not taking any oral hypoglycaemic agent or insulin. The average $\mathrm{HbA}_{1 \mathrm{c}}$ was $7.93 \pm 1.61 \%$. The clinical study was performed twice, once within 2 weeks before the bariatric procedure, and the 
second time 24 months later in the 2 weeks before plastic reconstruction of the abdominal wall. During surgery, abdominal muscle tissue biopsies were obtained from rectus abdominis muscles. The anthropometric, clinical and metabolic characteristics of the study patients are shown in Tables 1 and 2. The study protocol was approved by the Institutional Review Board and Ethics Committee of the Catholic University-Policlinico Gemelli (Rome, Italy).

$B P D$ This malabsorptive surgical procedure [23] consists of an approximately $60 \%$ distal gastric resection with stapled closure of the duodenal stump. The residual volume of the stomach is about $300 \mathrm{ml}$. The small bowel is transected at $2.5 \mathrm{~m}$ from the ileo-caecal valve and its distal end is anastomosed to the remaining stomach. The proximal end of the ileum, comprising the remaining small bowel (involved in carrying bilio-pancreatic juice but excluded from food transit), is anastomosed in an end-toside fashion to the bowel, $50 \mathrm{~cm}$ proximal to the ileo-caecal valve. Consequently, the total length of absorbing bowel is reduced to $250 \mathrm{~cm}$, the final $50 \mathrm{~cm}$ of which, the so-called common channel, represents the site where ingested food and bilio-pancreatic juices mix.

Body composition During the two study stages (before and after BPD), body weight was measured to the nearest $0.1 \mathrm{~kg}$ by a beam scale and height to the nearest $0.5 \mathrm{~cm}$ using a stadiometer (Holatin, Crosswell, UK). Waist circumference was measured just above the uppermost lateral border of the right ileum using the NHANES protocol [24]. Hip circumference was measured at the maximum extension of the buttocks as viewed from the side. Body composition was estimated by isotopic dilution $[25,26]$. Total body water was determined using $0.19 \mathrm{~Bq}$ of ${ }^{3} \mathrm{H}$-labelled water in $5 \mathrm{ml}$ of saline solution administered as an intravenous bolus injection. Blood samples were drawn before and $3 \mathrm{~h}$ after the injection. Radioactivity was determined in duplicate on $0.5 \mathrm{ml}$ of plasma using a beta-scintillation counter (1600TR; Canberra-Packard, Meriden, CT, USA). Corrections were made (5\%) for non-aqueous hydrogen exchange; water density at body temperature was assumed to be $0.99371 \mathrm{~kg} / \mathrm{l}$. Total body water $(\mathrm{kg})$ was computed as ${ }^{3} \mathrm{H}_{2} \mathrm{O}$ dilution space (litres) $\times 0.95 \times 0.99371$. The within-patient $\mathrm{CV}$ for this method is $1.5 \%$. Free-fat mass (FFM) (kg) was obtained by dividing total body water by 0.732 [25].

Euglycaemic-hyperinsulinaemic clamp procedure Peripheral insulin sensitivity was evaluated by a $2 \mathrm{~h}$ euglycaemichyperinsulinaemic clamp. The fasting plasma glucose concentration was maintained throughout the insulin infusion by means of a variable glucose infusion and blood glucose determinations every $5 \mathrm{~min}$. Whole-body glucose uptake $\left(M\right.$ value) in $\mu \mathrm{mol}\left(\mathrm{kg}_{\mathrm{FFM}}\right)^{-1} \mathrm{~min}^{-1}$ was determined during a primed constant infusion of insulin (at the rate of 6 pmol kg${ }^{-1} \min ^{-1}$ ). Value of whole-body glucose uptake was normalised for FFM. The quantitative insulin sensitivity check index (QUICKI) was calculated as reported by Katz et al. [27].

Table 1 Anthropometric and metabolic variables measured in NGT and type 2 diabetic patients before and after weight loss induced by BPD

\begin{tabular}{|c|c|c|c|c|c|c|c|}
\hline \multirow[t]{2}{*}{ Variables } & \multicolumn{3}{|l|}{ NGT } & \multicolumn{3}{|c|}{ Type 2 diabetes } & \multirow{2}{*}{$\begin{array}{l}\text { NGT vs type } 2 \text { diabetes } \\
p \text { value }\end{array}$} \\
\hline & Pre-surgery & Post-surgery & $p$ value & Pre-surgery & Post-surgery & $p$ value & \\
\hline$n$ & 11 & & & 10 & & & \\
\hline Sex (men/women) & $3 / 8$ & & & $3 / 7$ & & & \\
\hline Age (years) & $45 \pm 5$ & & & $48 \pm 6$ & & & \\
\hline Weight (kg) & $146.5 \pm 26.6$ & $83.36 \pm 13.5$ & 0.000001 & $146.5 \pm 66.3$ & $83.60 \pm 14.6$ & 0.000155 & 0.695 \\
\hline BMI $\left(\mathrm{kg} / \mathrm{m}^{2}\right)$ & $55.12 \pm 9.3$ & $31.28 \pm 3.3$ & 0.000002 & $53.41 \pm 5.4$ & $32.05 \pm 4.1$ & 0.000005 & 0.537 \\
\hline WHR & $1.00 \pm 0.08$ & $0.90 \pm 0.03$ & 0.000144 & $0.97 \pm 0.08$ & $0.89 \pm 0.03$ & 0.009776 & 0.665 \\
\hline Fat mass (kg) & $66.5 \pm 12.9$ & $21.00 \pm 5.3$ & 0.0000002 & $62.5 \pm 17.4$ & $21.3 \pm 5.2$ & 0.000033 & 0.769 \\
\hline FFM (kg) & $80.0 \pm 14.5$ & $62.4 \pm 10.2$ & 0.000071 & $79.0 \pm 11.0$ & $62.3 \pm 11.8$ & 0.000162 & 0.857 \\
\hline Fasting glucose $(\mathrm{mmol} / \mathrm{l})$ & $5.02 \pm 0.32$ & $4.03 \pm 0.14$ & 0.000001 & $7.96 \pm 2.78$ & $4.11 \pm 0.20$ & 0.001662 & 0.670 \\
\hline Fasting insulin (pmol/l) & $89.4 \pm 22.8$ & $53.4 \pm 12.0$ & 0.009038 & $136.8 \pm 87.6$ & $44.4 \pm 16.8$ & 0.011728 & 0.437 \\
\hline Cholesterol (mmol/1) & $5.63 \pm 0.9$ & $3.9 \pm 0.6$ & 0.000012 & $5.48 \pm 2.06$ & $3.78 \pm 1.1$ & 0.041392 & 0.683 \\
\hline HDL-cholesterol (mmol/l) & $0.99 \pm 0.07$ & $1.25 \pm 0.15$ & 0.000035 & $1.06 \pm 0.4$ & $1.28 \pm 0.13$ & 0.001150 & 0.898 \\
\hline Triacylglycerol (mmol/l) & $2.05 \pm 0.3$ & $1.38 \pm 0.22$ & 0.000041 & $2.16 \pm 1.07$ & $1.58 \pm 0.55$ & 0.177666 & 0.269 \\
\hline
\end{tabular}

Data are means \pm standard deviation

Statistical analyses comparing pre-surgery and post-surgery state were performed by the paired $t$ test. A comparison between NGT and type 2 diabetic patients was performed using ANCOVA, with $p$ values reported in the last column on the right

Values significant at $p<0.05$ 
Table 2 Insulin sensitivity and dynamic metabolic variables of the patients

\begin{tabular}{|c|c|c|c|c|c|c|c|}
\hline \multirow[t]{2}{*}{ Variables } & \multicolumn{3}{|l|}{ NGT } & \multicolumn{3}{|l|}{ Type 2 diabetes } & \multirow{2}{*}{$\begin{array}{l}\text { NGT vs type } 2 \\
\text { diabetes } \\
p \text { value }\end{array}$} \\
\hline & Pre-surgery & Post-surgery & $p$ value & Pre-surgery & Post-surgery & $p$ value & \\
\hline $\begin{array}{l}M \text { value }(\mu \mathrm{mol} \\
{\left[\mathrm{kg} \mathrm{FFM}^{-1}\right.} \\
\left.\min ^{-1}\right)\end{array}$ & $11.37 \pm 1.72$ & $42.07 \pm 5.72$ & 0.000000007 & $13.43 \pm 2.05$ & $41.01 \pm 5.38$ & 0.00000003 & 0.395 \\
\hline QUICKI index & $0.32 \pm 0.01$ & $0.36 \pm 0.01$ & 0.0039 & $0.29 \pm 0.02$ & $0.37 \pm 0.03$ & 0.000065 & 0.383 \\
\hline $\begin{array}{l}\text { Insulin during } \\
\text { clamp (pmol/1) }\end{array}$ & $590.28 \pm 52.2$ & $555.3 \pm 26.3$ & 0.0865 & $552.54 \pm 56.7$ & $552.0 \pm 28.0$ & 0.98 & 0.792 \\
\hline $\begin{array}{l}\text { Fasting glucose } \\
\text { oxidation }(\mu \mathrm{mol} \\
{\left[\mathrm{kg} \mathrm{FFM}^{-1}\right.} \\
\left.\min ^{-1}\right)\end{array}$ & $5.11 \pm 4.4$ & $15.99 \pm 9.99$ & 0.008812 & $4.99 \pm 2.9$ & $8.27 \pm 3.6$ & 0.011854 & 0.038 \\
\hline $\begin{array}{l}\text { Fasting lipid } \\
\text { oxidation (mg } \\
{[\mathrm{kg} \mathrm{FFM}]^{-1}} \\
\left.\min ^{-1}\right)\end{array}$ & $1.57 \pm 0.49$ & $0.89 \pm 0.46$ & 0.020589 & $1.98 \pm 0.32$ & $1.51 \pm 0.34$ & 0.000176 & 0.005 \\
\hline $\begin{array}{l}\text { Glucose oxidation } \\
\text { during clamp } \\
(\mu \mathrm{mol}[\mathrm{kg} \\
\text { FFM }]^{-1} \\
\left.\min ^{-1}\right)\end{array}$ & $13.26 \pm 4.77$ & $23.19 \pm 8.1$ & 0.001440 & $13.26 \pm 5.1$ & $19.98 \pm 7.4$ & 0.040453 & 0.349 \\
\hline $\begin{array}{l}\text { Lipid oxidation } \\
\text { during the clamp } \\
\left(\mathrm{mg}[\mathrm{kg} \mathrm{FFM}]^{-1}\right. \\
\left.\min ^{-1}\right)\end{array}$ & $0.10 \pm 0.04$ & $0.04 \pm 0.03$ & 0.002738 & $0.14 \pm 0.03$ & $0.16 \pm 0.05$ & 0.215055 & 0.00006 \\
\hline Fasting RQ & $0.77 \pm 0.05$ & $0.85 \pm 0.07$ & 0.012338 & $0.76 \pm 0.02$ & $0.79 \pm 0.03$ & 0.000773 & 0.023 \\
\hline RQ during clamp & $0.83 \pm 0.03$ & $0.91 \pm 0.04$ & 0.000441 & $0.82 \pm 0.03$ & $0.84 \pm 0.04$ & 0.248183 & 0.001 \\
\hline$\Delta \mathrm{RQ}$ & $0.067 \pm 0.058$ & $0.061 \pm 0.071$ & 0.841087 & $0.061 \pm 0.029$ & $0.046 \pm 0.030$ & 0.340470 & 0.538 \\
\hline $\operatorname{REE}(\mathrm{kJ} / 24 \mathrm{~h})$ & $2487 \pm 624$ & $2078 \pm 252$ & 0.078091 & $2868 \pm 433$ & $2122 \pm 263$ & 0.000138 & 0.892 \\
\hline 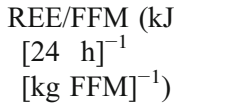 & $31.1 \pm 5.0$ & $34.2 \pm 6.9$ & 0.281711 & $36.4 \pm 3.1$ & $34.5 \pm 3.4$ & 0.214784 & 0.776 \\
\hline
\end{tabular}

Data are means \pm standard deviation

Statistical analyses comparing pre-surgery and post-surgery state were performed by paired $t$ tests. A comparison between NGT and type 2 diabetic patients was performed using ANCOVA, with $p$ values reported in last column on right

Values significant at $p<0.05$

Analytical procedures See Electronic supplementary material (ESM).

Substrate oxidation studies The patients spent a day (starting at 08.00 hours) in the respiratory chamber of the Metabolism Unit of the Catholic University School of Medicine in Rome, after an overnight fast. The characteristics of the device have been described previously [28]. All patients were assigned a diet with an energy content reflecting their habitually consumed diet. The food given and returned was weighed to the nearest gram on precision scales (KS-01; Rowenta, Berlin, Germany). Resting energy expenditure (REE) and respiratory quotient (RQ) were calculated from oxygen consumption, carbon dioxide production and nitrogen urinary excretion according to standard formulas [28]. Results are presented as $\mathrm{kJ} / 24 \mathrm{~h}$ and after normalisation to FFM (kJ $[24 \mathrm{~h}]^{-1}[\mathrm{~kg} \mathrm{FFM}]^{-1}$ ). Indirect calorimetry [29] was used to determine $\mathrm{O}_{2}$ consumption and $\mathrm{CO}_{2}$ production during the $30 \mathrm{~min}$ preceding the clamp and in the last $80 \mathrm{~min}$ of the clamp (in steady-state condition) to estimate net rates of carbohydrate and lipid oxidation (SensorMedics 2900; SensorMedics, Yorba Linda, CA, USA). Non-oxidative glucose disposal rate was calculated as total glucose disposal rate minus glucose oxidation rate.

\section{$R N A$ extraction and real-time PCR analysis See ESM.}

Statistical analysis Data analyses were performed with SPSS statistical software (SPSS, Chicago, IL, USA). Data are reported as mean $\pm \mathrm{SD}$, unless otherwise specified. 
Paired and unpaired $t$ tests were performed to compare data from the same patients before and after BPD and data of NGT and type 2 diabetic patients after BPD.

To assess differences between the two groups, analysis of covariance (ANCOVA) was performed. The dependent variable was the delta value (calculated as the value before the surgery minus the value after BPD), the fixed factor was the time (before BPD) and the covariate used in the analysis was the baseline value for the variable under investigation. Predictors of $M F N 2$ and $P G C-1 \beta$ mRNA level changes were tested using the Pearson correlation. Distribution statistics for the residuals were calculated to check whether assumptions of normality were met (i.e. skewness and kurtosis $<2.0$ ). Multiple linear regression analysis was then used to fit models to predict the determinants of fasting glucose and fasting lipid oxidation. Variables were allowed to enter the models if significant at the $<0.05$ probability level.

\section{Results}

Anthropometric and plasma variables in normal glucosetolerant or type 2 diabetic morbidly obese patients before and after BPD Table 1 shows anthropometric and body composition data, measured before and 2 years after BPD, in obese NGT and obese type 2 diabetic patients. After BPD, BMI significantly decreased in both groups, with concomitant reductions in fat mass (68.4 and $65.9 \%$, respectively) and FFM (22 and 21.1\%, respectively). As to metabolic variables, BPD significantly decreased plasma levels of glucose, insulin, triacylglycerol and cholesterol, while HDLcholesterol levels increased after surgery in both groups (Table 1). As shown in Table 1, ANCOVA analysis showed no statistically significant differences between groups.

Morbidly obese NGT and type 2 diabetic patients show improved insulin sensitivity but a different substrate oxidation profile in response to BPD BPD resulted in the normalisation of insulin sensitivity. As reported in Table 2, whole-body glucose disposal ( $M$ value) significantly increased both in NGT (from $11.38 \pm 1.72$ to $42.07 \pm$ $5.72 \mu \mathrm{mol}[\mathrm{kg} \mathrm{FFM}]^{-1} \mathrm{~min}^{-1}$ ) and type 2 diabetic patients (from $13.43 \pm 2.05$ to $41.01 \pm 5.38 \mu \mathrm{mol}[\mathrm{kg} F]^{-1}$ $\left.\min ^{-1}\right)$. No differences in $M$ values were detected in NGT and in diabetic patients, which is in keeping with previous reports [14, 30]. Plasma insulin concentrations during the clamp were similar in all patients studied (Table 2). We also calculated the QUICKI index as a surrogate of hepatic insulin sensitivity [31]. The QUICKI index was significantly increased after BPD, both in type 2 diabetic and in NGT patients (Table 2). Altogether these data suggest that BPD causes a net improvement not only of glucose disposal mediated by peripheral insulin, but also of hepatic insulin sensitivity.

However, NGT and type 2 diabetic patients showed a different capacity to oxidise substrates under fasting conditions and during the clamp. After BPD, both groups increased glucose oxidation during fasting but the increase was much greater in NGT than in diabetic patients (3.1- and 1.6-fold respectively). Conversely, both groups showed decreased lipid oxidation during fasting, the effects being greater in NGT than in diabetic patients (44 and 24\% decrease respectively). ANCOVA analysis provided further evidence that the changes induced by BPD in fasting glucose and lipid oxidation were significantly different in NGT and type 2 diabetes patients $(p=0.038$ and $p=0.005$, respectively) (Table 2). During the clamp, glucose oxidation markedly increased in response to BPD in NGT and type 2 diabetic patients, while lipid oxidation decreased in the former but did not change in the latter after BPD (Table 2). These results again highlight a different capacity of the two groups (NGT and type 2 diabetics) to oxidise lipids during the clamp $(p<0.0001)$.

The respiratory quotient at fasting and during the clamp in the post-surgery state was markedly lower in diabetic patients than in NGT patients (Table 2), indicating that diabetic patients oxidise more lipids. $\triangle \mathrm{RQ}$ values were not statistically different in the two groups (Table 2). Neither the change in REE nor that in REE/FFM before and after BPD were different between the two groups (Table 2).

Overall, these data indicate that the effect of BPD in terms of substrate utilisation, whether either fasting or insulin-mediated, is larger in NGT patients.

Morbidly obese type 2 diabetic patients show impaired muscle capacity to induce expression of nuclear genes encoding mitochondrial proteins in response to BPD To further investigate the molecular basis for the differential metabolic adaptations of NGT and type 2 diabetic patients to BPD, and also because it has been reported that genes involved in mitochondrial function are induced in response to weight loss in morbidly obese patients [18], we studied the expression of genes encoding mitochondrial proteins. To this end, abdominal skeletal muscle biopsies were collected before and after BPD and used for gene expression analysis. In particular, real-time PCR analysis was used to evaluate mRNA levels of a marker of mitochondrial fusion that regulates mitochondrial activity (MFN2), two genes that encode for constitutive proteins (PORIN [also known as VDAC1] and CS) and a gene encoded by mitochondrial DNA (COXIII). As shown in Fig. 1, BPD resulted in a significant increase of $M F N 2, C S$ and PORIN mRNA levels in NGT patients (1.63-, 2.45- and 2.99-fold increase, respectively). In contrast, MFN2 expres- 
sion significantly decreased in type 2 diabetic patients (by 0.48 -fold) and no changes were detected in CS or PORIN expression in the type 2 diabetic group after surgery (Fig. 1). COXIII transcripts levels were not modulated by BPD either in the NGT or in the type 2 diabetic group (Fig. 1).

These results indicate a fundamental difference between NGT and type 2 diabetic patients in the regulation of nuclear expression of genes encoding mitochondrial proteins. Thus NGT patients experienced induction of MFN2, PORIN or CS, whereas type 2 diabetic patients had no capacity to do so.

Morbidly obese type 2 diabetic patients show impaired muscle capacity to induce gene expression of $P G C-1 \alpha$, $P G C-1 \beta$ and SIRT1 in response to BPD Next, we studied whether BPD affects the expression of genes encoding transcriptional regulators of mitochondrial function. Hence, mRNA levels of $P G C-1 \beta, P G C-1 \alpha, E R R \alpha$ (also known as ESRRA) and PPAR $\delta$ in abdominal skeletal muscle were measured before and after BPD. As shown in Fig. 2, $P G C-1 \beta$ mRNA levels significantly increased after surgery (2.04-fold) in the NGT group, but not in type 2 diabetic patients. $P G C-1 \alpha$ and PPAR $\delta$ mRNA levels increased (1.53- and 1.75-fold respectively; differences not significant) in NGT patients, but not in diabetic patients in response to BPD and as a result marked differences were detected between NGT and diabetic patients after surgery (Fig. 3). No differences were observed in the expression of $E R R \alpha$ in either group after BPD (Fig. 2).

SIRT1, a NAD ${ }^{+}$-dependent deacetylase, regulates $P G C$ $1 \alpha$ activity in liver and muscle tissues [21, 22]. Based on the observation that muscle $P G C-1 \alpha$ is induced in NGT patients but not in type 2 diabetic patients after BPD, we analysed whether SIRT1 participates in the differential gene expression pattern detected in the two groups. In keeping with previous data, SIRT1 mRNA levels significantly increased after BPD (2.27-fold) in the NGT group, but not in the diabetic group (Fig. 3).

Substrate oxidation differentially correlates with gene expression in NGT and type 2 diabetic patients To gain more insights into the different capacity of the NGT group to oxidise substrates compared with diabetic patients, correlation analyses between glucose and lipid oxidation during fasting with the levels of the different transcripts (before and after BPD) were performed. Pearson's correlation results for NGT and diabetic patients are reported in Table 3. PPAR- $\delta, E R R-\alpha$ and SIRT1 mRNA levels did not correlate with any of the variables under study (data not shown). Interestingly, MFN2 mRNA levels positively correlated with fasting glucose oxidation and negatively with fasting lipid oxidation in the NGT group. In contrast, MFN2 mRNA levels did not correlate with any of the above-mentioned variables in type 2 diabetic patients. Similarly, the expression of CS, PORIN, PGC-1 $\alpha$ or $P G C-1 \beta$ positively correlated with fasting glucose oxidation in NGT patients (Table 3). Analysis of delta values also revealed a positive correlation between the expression of genes $(C S$ or $P G C-1 \alpha)$ and fasting glucose oxidation in NGT, but not in diabetic patients (Table 3).

A stepwise linear regression analysis was performed in the NGT group, including in the model MFN2, CS, PORIN, $P G C-1 \alpha$ and $P G C-1 \beta$ as independent variables, and fasting glucose oxidation as the dependent variable. MFN2 expression turned out to be the main predictor of fasting glucose oxidation $\left(R^{2}=0.496, p=0.001\right)$, as shown in Fig. 4a. Such correlations, however, were lost in the diabetic group, as illustrated by the scatter plot in Fig. $4 \mathrm{~b}$. The expression of CS, PORIN, PGC-1 $\alpha$ or $P G C-1 \beta$ did not predict glucose oxidation (data not shown).

$C S$ expression was a good predictor of fasting lipid oxidation $\left(R^{2}=0.311, p=0.009\right)$, whereas MFN2 or PORIN expression were not determinants of lipid oxidation (data not shown). In all, our data indicate that the modulation of
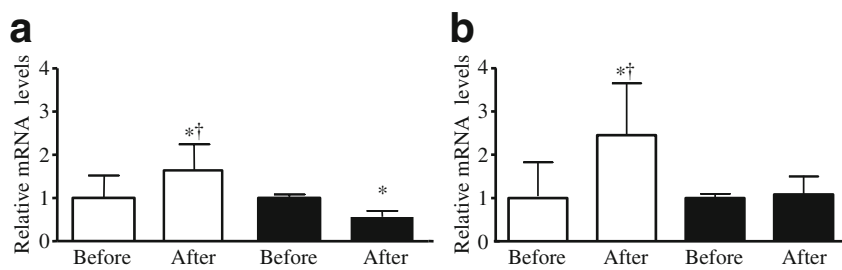

Fig. 1 Skeletal muscle gene expression of mitochondrial proteins in NGT (white bars) and type 2 diabetic patients (black bars) in response to BPD. Real-time PCR was performed in abdominal skeletal muscle biopsies from NGT and type 2 diabetic patients before and 2 years after BPD surgery. a MFN2; ${ }^{*} p=0.0311, \uparrow p=0.0003$ in NGT; ${ }^{*} p=$ 0.00003 in type 2 diabetic; b $C S ;{ }^{*} p=0.02, \dagger p=0.0083$; c PORIN;
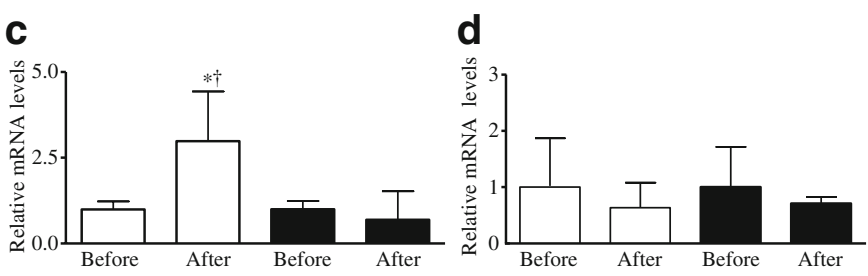

${ }^{*} p=0.033, \dagger p=0.034$; and $\mathbf{d}$ COXIII. Data are means \pm standard deviation. Statistical analyses comparing pre-surgery and post-surgery state were performed by paired $t$ test $(*)$. Statistical analyses comparing post-surgery between NGT and type 2 diabetic patients were performed by unpaired $t$ test $(\dagger)$ 
a

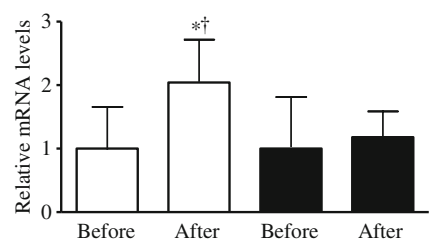

b

Fig. 2 Skeletal muscle gene expression of transcriptional regulators of mitochondrial function in NGT (white bars) and type 2 diabetic patients (black bars) in response to BPD. Real-time PCR was performed as in Fig. 1. a $P G C-1 \beta ; * p=0.046, \dagger p=0.049$; b $P G C$ $1 \alpha ; \dagger p=0.048$; $\mathbf{c} P P A R-\delta ; \dagger p=0.045$; and $\mathbf{d} E R R \alpha$. Data are means \pm

glucose oxidation after BPD in NGT patients seems to be dependent upon changes in MFN2 expression.

\section{Discussion}

A major finding of this study is the differential pattern of changes in the expression of genes involved in the regulation of mitochondrial biogenesis/activity in skeletal muscle from morbidly obese type 2 diabetic and NGT patients in response to BPD. Thus, whereas the expression of some mitochondrial genes, including MFN2, PORIN or $C S$, of mitochondrial regulatory genes such as $P G C-1 \beta$, $P G C-1 \alpha, P P A R-\delta$ or SIRT1 increased in the skeletal muscle of NGT patients 2 years after BPD, no changes were detected in type 2 diabetic patients. This pattern of changes was parallel to a differential oxidative profile: whereas glucose oxidation during fasting was higher in the NGT group after BPD, lipid oxidation levels (both in the fasting state and during the euglycaemic-hyperinsulinaemic clamp) were higher in the type 2 diabetic group after BPD. The BPD-induced improvement of insulin sensitivity, as shown by insulin-mediated glucose uptake and oxidation, was comparable in both patient groups. This suggests that improvement of insulin sensitivity is independent of the

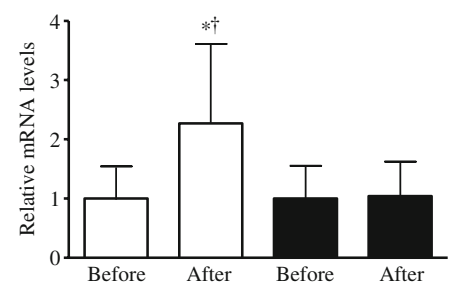

Fig. 3 SIRT1 gene expression is upregulated in the abdominal skeletal muscle of NGT (white bars) as compared with type 2 diabetic patients (black bars) after BPD. Data are means \pm standard deviation. Statistical analyses comparing pre-surgery and post-surgery state were performed by the paired $t$ test $(*)$. Statistical analyses comparing postsurgery between NGT and type 2 diabetic patients were performed by the unpaired $t$ test $(\dagger) .{ }^{*} p=0.049, \dagger p=0.020$
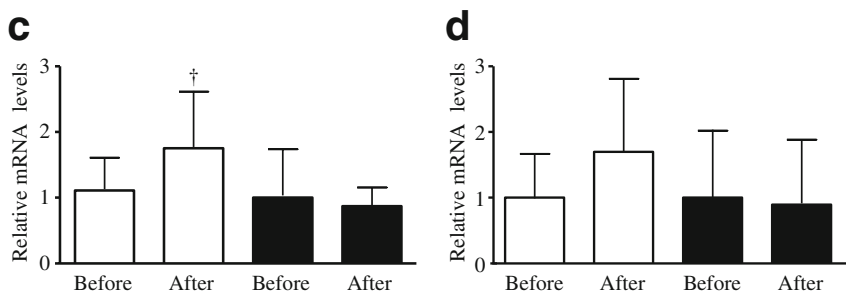

standard deviation. Statistical analyses comparing pre-surgery and post-surgery state were performed by the paired $t$ test $(*)$. Statistical analyses comparing post-surgery between NGT and type 2 diabetic patients were performed by the unpaired $t$ test $(\dagger)$

expression of genes involved in mitochondrial activity, and therefore the mechanisms of increased insulin sensitivity triggered by BPD are of a different nature.

The pattern of changes detected in response to BPD consists of enhanced expression of genes encoding functional and regulatory components of mitochondria such as CS, PORIN or MFN2, as well as of genes encoding regulators of mitochondrial biogenesis such as $P G C \mathrm{~s}$, $E R R \alpha$ and activators of mitochondrial function such as $P P A R \delta$. We propose that the physiological purpose of the pattern of changes in gene expression detected in skeletal muscle in response to BPD is to trigger mitochondrial biogenesis in skeletal muscle, which favours increased glucose oxidation, under conditions of reduced lipid availability. This needs to be confirmed by direct analysis in muscle, but is consistent with the observations that whole-body glucose oxidation was increased. Patients undergoing BPD have reduced lipid availability provided that BPD causes lipid malabsorption. That is probably the reason why we found lower whole-body lipid oxidation after BPD in NGT patients.

Our study shows some limitations, namely: (1) diet was not controlled in the two groups of patients and patients did not follow any specific dietary regimen or have any dietary restrictions; (2) the patients had a sedentary lifestyle either before or after BPD, but this was not controlled; and (3) only gene expression was monitored, so there is a need to determine levels of proteins involved in mitochondrial biogenesis/function in skeletal muscle.

Mitofusin-2 (MFN2) is a mitochondrial fusion protein that regulates mitochondrial metabolism $[19,20]$. MFN2 loss of function reduces glucose oxidation and mitochondrial membrane potential in muscle and non-muscle cells $[19,20]$. Similarly, MFN2 gain of function enhances glucose oxidation and mitochondrial membrane potential [20]. The mechanisms are only partially known and seem to be dependent on the expression of subunits of the oxidative phosphorylation system [20]. In this study we have demonstrated that MFN2 is upregulated in skeletal muscle from morbidly obese NGT patients in response to BPD, 
Table 3 Correlation coefficients between different variables in NGT and type 2 diabetic patients for glucose and lipid oxidation

\begin{tabular}{|c|c|c|c|c|c|c|c|c|}
\hline \multirow[t]{2}{*}{ Variables } & \multicolumn{2}{|l|}{ NGT } & \multicolumn{2}{|l|}{$\operatorname{NGT}(\Delta)$} & \multicolumn{2}{|l|}{$\mathrm{T} 2 \mathrm{D}$} & \multicolumn{2}{|l|}{$\mathrm{T} 2 \mathrm{D}(\Delta)$} \\
\hline & Pearson's $r$ & $p$ value & Spearman's $\rho$ & $p$ value & Pearson's $r$ & $p$ value & Spearman's $\rho$ & $p$ value \\
\hline \multicolumn{9}{|c|}{$\begin{array}{l}\text { Fasting glucose oxidation (mRNA } \\
\text { levels) }\end{array}$} \\
\hline MFN2 & 0.675 & 0.001 & 0.545 & 0.083 & -0.308 & 0.247 & -0.119 & 0.779 \\
\hline$C S$ & 0.549 & 0.010 & 0.673 & 0.033 & 0.634 & 0.004 & 0.001 & 0.998 \\
\hline$P G C-1 \beta$ & 0.521 & 0.019 & 0.367 & 0.332 & 0.037 & 0.882 & 0.001 & 0.998 \\
\hline PORIN & 0.480 & 0.028 & 0.333 & 0.347 & 0.156 & 0.524 & -0.183 & 0.637 \\
\hline$P G C-1 \alpha$ & 0.473 & 0.031 & 0.794 & 0.006 & 0.025 & 0.920 & 0.617 & 0.077 \\
\hline \multicolumn{9}{|c|}{ Fasting lipid oxidation (mRNA levels) } \\
\hline$M F N 2$ & -0.586 & 0.040 & -0.673 & 0.023 & 0.328 & 0.215 & -0.048 & 0.911 \\
\hline$C S$ & -0.558 & 0.009 & -0.539 & 0.108 & -0.424 & 0.070 & -0.083 & 0.831 \\
\hline PORIN & -0.477 & 0.029 & -0.079 & 0.829 & -0.033 & 0.895 & -0.250 & 0.516 \\
\hline
\end{tabular}

Correlations between variables were calculated in the total populations (NGT or type 2 diabetes) with Pearson's correlation coefficient and as delta values (pre-BPD - post-BPD) using Spearman's correlation coefficient, respectively. $r$ values are reported

Values significant at $p<0.05$

which correlates positively with glucose oxidation. This is in keeping with previous findings observed in women subjected to BPD [18] or in patients in whom weight loss was induced by Roux-en-Y gastric bypass [32]. Our data additionally indicate that $M F N 2$ expression is a good predictor of fasting glucose oxidation in NGT patients, much better than the expression of genes encoding for regulators of mitochondrial biogenesis such as $P G C-1 \alpha$ or $P G C-1 \beta$, and also better than the expression of genes encoding for constitutive proteins such as porin or citrate synthase (and therefore markers of mitochondrial mass). MFN2 induction was not detected in skeletal muscle from type 2 diabetic patients in response to BPD (in fact, a reduction was observed) and these patients also showed very small changes in substrate oxidation. In all, our results further support the view that $M F N 2$ expression is a positive regulator of glucose oxidation.

The nuclear transcription factor $P P A R \delta$ and the nuclear co-activators $P G C-1 \alpha$ or $P G C-1 \beta$ regulate mitochondrial function and/or mitochondrial biogenesis in muscle [33]. Similarly, both regulate MFN2 transcription through activation of $E R R-\alpha$ [34]. Based on these observations, we studied whether expression of $P G C-1 \alpha, P G C-1 \beta$ or PPAR- $\delta$ may explain the regulation of MFN2, PORIN or $C S$ in skeletal muscle from morbidly obese patients after BPD. $P G C-1 \alpha, P G C-1 \beta$ and $P P A R-\delta$, as well as SIRT1, a modulator of $P G C-1 \alpha$ [21, 22], were upregulated in NGT patients after BPD. In contrast, the expression of $P G C-1 \alpha$, $P G C-1 \beta, P P A R-\delta$ and SIRT1, as well as that of those genes encoding mitochondrial proteins, was unaltered in morbidly obese type 2 diabetic patients in response to BPD, despite massive weight loss and normalisation of insulin sensitivity.

Our data indicate that BPD triggers a complex set of events (perhaps due to malabsorption, reduced food intake, partial loss-of-function of the intestinal tract induced by BPD) that induces genes critical to mitochondrial function such as $P G C-1 \alpha, P G C-1 \beta, P P A R-\delta$; as a result, Mfn 2
Fig. 4 MFN2 mRNA levels in the abdominal skeletal muscle correlated with fasting glucose oxidation in the NGT group. Simple scatterplots of the correlations between MFN2 mRNA expression and fasting glucose oxidation $\mathbf{a}$ in the NGT group $(y=-0.3164+1.6908 x)$ and b in type 2 diabetic patients $(y=1.7708-0.7494 x)$. Lines indicate the best fit linear regression
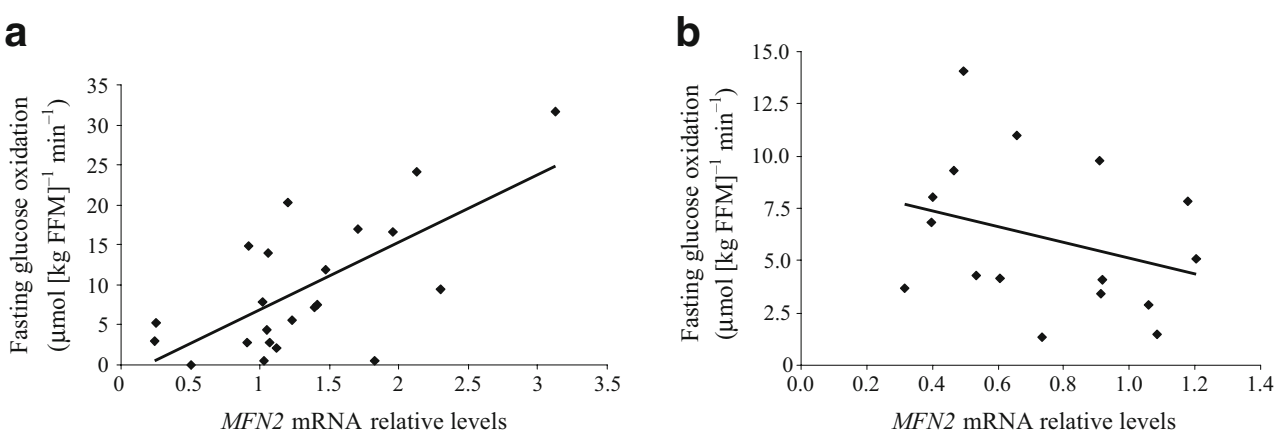
expression and glucose oxidation are concomitantly enhanced. Increased $P G C-1 \alpha$ expression has also been reported in muscle from obese patients after weight loss induced by Roux-en-Y gastric bypass [32]. In contrast, $P G C-1 \alpha, P G C-1 \beta, P P A R-\delta$ and SIRT1 were not induced in morbidly obese type 2 diabetic patients in response to BPD, which explains the lack of stimulation of genes encoding for mitochondrial proteins (citrate synthase, porin or MFN2) and the blunted changes of glucose and lipid oxidation particularly under fasting conditions.

In different insulin-resistant states, including type 2 diabetes, a defective mitochondrial function has been reported in skeletal muscle [1, 2, 35-37]. Muscle mitochondria from patients with type 2 diabetes show reduced size, reduced activity of the electron transport chain and reduced ADP-stimulated and maximal mitochondrial respiratory capacity $[1,36,37]$. In parallel, type 2 diabetes is associated with reduced expression of genes of oxidative metabolism as well as with repression of MFN2 [38-40]. In skeletal muscle of non-diabetic individuals with a family history of type 2 diabetes, decreased expression of nuclear genes encoding proteins of oxidative phosphorylation has been reported [39, 40], along with reduced in vivo oxidative phosphorylation [35, 41]. However, the role of mitochondria in the pathogenesis of insulin resistance and diabetes is still unclear. In vastus lateralis muscles from obese insulin-resistant patients after weight loss, no increase in oxidative enzyme activities was reported, in spite of an improvement of insulin sensitivity [42]. In addition, Toledo and collaborators [37] have reported that weight loss, without physical exercise, in obese patients ameliorates insulin sensitivity with no effects on muscle mitochondrial capacity.

Our findings suggest that weight loss consequent to BPD exerts a beneficial effect on insulin sensitivity via mechanisms that are independent of skeletal muscle expression of genes involved in mitochondrial biogenesis/function. Furthermore, the observation that gene expression is not altered with weight loss in type 2 diabetic patients while it is induced in patients with NGT suggests the presence of a heritable component.

Acknowledgements This study was supported by research grants from the Ministerio de Educación y Cultura (SAF 2005-00445 and SAF 2008-03803), by grant 2005SGR00947 from the Generalitat de Catalunya, and a grant from CIBERDEM (Instituto de Salud Carlos III). M. I. Hernandez-Alvarez is the recipient of a predoctoral fellowship from the CONACYT, Mexico. M. Liesa is the recipient of a predoctoral fellowship from the Ministerio de Educación y Cultura, Spain. A. Zorzano is the recipient of a Science Intensification Award from the University of Barcelona. We thank T. Yates for editorial support.

Duality of interest The authors declare that there is no duality of interest associated with this manuscript.

\section{References}

1. Kelley DE, He J, Menshikova EV, Ritov VB (2002) Dysfunction of mitochondria in human skeletal muscle in type 2 diabetes. Diabetes 51:2944-2950

2. Petersen KF, Befroy D, Dufour S et al (2003) Mitochondrial dysfunction in the elderly: possible role in insulin resistance. Science 300:1140-1142

3. Holloszy JO (2009) Skeletal muscle "mitochondrial deficiency" does not mediate insulin resistance. Am J Clin Nutr 89:463S$466 \mathrm{~S}$

4. Mogensen M, Sahlin K, Fernstrom M et al (2007) Mitochondrial respiration is decreased in skeletal muscle of patients with type 2 diabetes. Diabetes 56:1592-1599

5. Ritov VB, Menshikova EV, He J, Ferrell RE, Goodpaster BH, Kelley DE (2005) Deficiency of subsarcolemmal mitochondria in obesity and type 2 diabetes. Diabetes $54: 8-14$

6. Boushel R, Gnaiger E, Schjerling P, Skovbro M, Kraunsoe R, Dela F (2007) Patients with type 2 diabetes have normal mitochondrial function in skeletal muscle. Diabetologia 50:790-796

7. Nair KS, Bigelow ML, Asmann YW et al (2008) Asian Indians have enhanced skeletal muscle mitochondrial capacity to produce ATP in association with severe insulin resistance. Diabetes 57:1166-1175

8. De Feyter HM, van den Broek NM, Praet SF, Nicolay K, van Loon LJ, Prompers JJ (2008) Early or advanced stage type 2 diabetes is not accompanied by in vivo skeletal muscle mitochondrial dysfunction. Eur J Endocrinol 158:643-653

9. Ross R (2003) Does exercise without weight loss improve insulin sensitivity? Diabetes Care 26:944-945

10. Schneider SH, Khachadurian AK, Amorosa LF, Clemow L, Ruderman NB (1992) Ten-year experience with an exercisebased outpatient life-style modification program in the treatment of diabetes mellitus. Diabetes Care 15:1800-1810

11. Buchwald H, Avidor Y, Braunwald E et al (2004) Bariatric surgery: a systematic review and meta-analysis. JAMA 292:17241737

12. Castagneto M, De Gaetano A, Mingrone G et al (1994) Normalization of insulin sensitivity in the obese patient after stable weight reduction with biliopancreatic diversion. Obes Surg 4:161-168

13. Greco AV, Mingrone G, Giancaterini A et al (2002) Insulin resistance in morbid obesity: reversal with intramyocellular fat depletion. Diabetes 51:144-151

14. Guidone C, Manco M, Valera-Mora E et al (2006) Mechanisms of recovery from type 2 diabetes after malabsorptive bariatric surgery. Diabetes 55:2025-2031

15. Gniuli D, Rosa G, Manco M et al (2005) Changes in fat mass influence SREBP-1c and UCP-2 gene expression in formerly obese subjects. Obes Res 13:567-573

16. Mingrone G, Rosa G, Greco AV et al (2003) Intramyocitic lipid accumulation and SREBP-1c expression are related to insulin resistance and cardiovascular risk in morbid obesity. Atherosclerosis 170:155-161

17. Mingrone G, Rosa G, Greco AV et al (2003) Decreased uncoupling protein expression and intramyocytic triglyceride depletion in formerly obese subjects. Obes Res 11:632-640

18. Mingrone G, Manco M, Calvani M, Castagneto M, Naon D, Zorzano A (2005) Could the low level of expression of the gene encoding skeletal muscle mitofusin-2 account for the metabolic inflexibility of obesity? Diabetologia 48:2108-2114

19. Bach D, Pich S, Soriano FX et al (2003) Mitofusin-2 determines mitochondrial network architecture and mitochondrial metabolism. A novel regulatory mechanism altered in obesity. J Biol Chem 278:17190-17197 
20. Pich S, Bach D, Briones P et al (2005) The Charcot-Marie-Tooth type 2A gene product, Mfn2, up-regulates fuel oxidation through expression of OXPHOS system. Hum Mol Genet 14:1405-1415

21. Gerhart-Hines Z, Rodgers JT, Bare O et al (2007) Metabolic control of muscle mitochondrial function and fatty acid oxidation through SIRT1/PGC-1alpha. EMBO J 26:1913-1923

22. Rodgers JT, Lerin C, Haas W, Gygi SP, Spiegelman BM, Puigserver $\mathrm{P}$ (2005) Nutrient control of glucose homeostasis through a complex of PGC-1alpha and SIRT1. Nature 434:113-118

23. Scopinaro N, Gianetta E, Civalleri D, Bonalumi U, Bachi V (1979) Bilio-pancreatic bypass for obesity: II. Initial experience in man. Br J Surg 66:618-620

24. Rodriguez BL, Fujimoto WY, Mayer-Davis EJ et al (2006) Prevalence of cardiovascular disease risk factors in U.S. children and adolescents with diabetes: the SEARCH for diabetes in youth study. Diabetes Care 29:1891-1896

25. Bonora E, Del Prato S, Bonadonna RC et al (1992) Total body fat content and fat topography are associated differently with in vivo glucose metabolism in nonobese and obese nondiabetic women. Diabetes 41:1151-1159

26. Culebras JM, Fitzpatrick GF, Brennan MF, Boyden CM, Moore FD (1977) Total body water and the exchangeable hydrogen. II. A review of comparative data from animals based on isotope dilution and desiccation, with a report of new data from the rat. Am J Physiol 232:R60-R65

27. Katz A, Nambi SS, Mather K et al (2000) Quantitative insulin sensitivity check index: a simple, accurate method for assessing insulin sensitivity in humans. J Clin Endocrinol Metab 85:24022410

28. Granato L, Brandes A, Bruni C, Greco AV, Mingrone G (2004) $\mathrm{VO} 2, \mathrm{VCO} 2$, and $\mathrm{RQ}$ in a respiratory chamber: accurate estimation based on a new mathematical model using the Kalman-Bucy method. J Appl Physiol 96:1045-1054

29. Ferrannini E (1988) The theoretical bases of indirect calorimetry: a review. Metabolism 37:287-301

30. Mingrone G, Manco M, Granato L et al (2005) Leptin pulsatility in formerly obese women. FASEB J 19:1380-1382

31. Hoffman RP (2008) Indices of insulin action calculated from fasting glucose and insulin reflect hepatic, not peripheral, insulin sensitivity in African-American and Caucasian adolescents. Pediatr Diabetes 9:57-61

32. Gastaldi G, Russell A, Golay A et al (2007) Upregulation of peroxisome proliferator-activated receptor gamma coactivator gene (PGC1A) during weight loss is related to insulin sensitivity but not to energy expenditure. Diabetologia 50:2348-2355

33. Puigserver P, Wu Z, Park CW, Graves R, Wright M, Spiegelman BM (1998) A cold-inducible coactivator of nuclear receptors linked to adaptive thermogenesis. Cell 92:829-839

34. Soriano FX, Liesa M, Bach D, Chan DC, Palacin M, Zorzano A (2006) Evidence for a mitochondrial regulatory pathway defined by peroxisome proliferator-activated receptor-gamma coactivator1 alpha, estrogen-related receptor-alpha, and mitofusin 2. Diabetes 55:1783-1791

35. Petersen KF, Dufour S, Befroy D, Garcia R, Shulman GI (2004) Impaired mitochondrial activity in the insulin-resistant offspring of patients with type 2 diabetes. N Engl J Med 350:664-671

36. Phielix E, Schrauwen-Hinderling VB, Mensink M et al (2008) Lower intrinsic ADP-stimulated mitochondrial respiration underlies in vivo mitochondrial dysfunction in muscle of male type 2 diabetic patients. Diabetes 57:2943-2949

37. Toledo FG, Menshikova EV, Azuma K et al (2008) Mitochondrial capacity in skeletal muscle is not stimulated by weight loss despite increases in insulin action and decreases in intramyocellular lipid content. Diabetes 57:987-994

38. Bach D, Naon D, Pich S et al (2005) Expression of Mfn2, the Charcot-Marie-Tooth neuropathy type $2 \mathrm{~A}$ gene, in human skeletal muscle: effects of type 2 diabetes, obesity, weight loss, and the regulatory role of tumor necrosis factor alpha and interleukin-6. Diabetes 54:2685-2693

39. Mootha VK, Lindgren CM, Eriksson KF et al (2003) PGC1alpha-responsive genes involved in oxidative phosphorylation are coordinately downregulated in human diabetes. Nat Genet 34:267-273

40. Patti ME, Butte AJ, Crunkhorn S et al (2003) Coordinated reduction of genes of oxidative metabolism in humans with insulin resistance and diabetes: potential role of PGC1 and NRF1. Proc Natl Acad Sci U S A 100:8466-8471

41. Morino K, Petersen KF, Dufour S et al (2005) Reduced mitochondrial density and increased IRS-1 serine phosphorylation in muscle of insulin-resistant offspring of type 2 diabetic parents. $\mathrm{J}$ Clin Invest 115:3587-3593

42. Simoneau JA, Veerkamp JH, Turcotte LP, Kelley DE (1999) Markers of capacity to utilize fatty acids in human skeletal muscle: relation to insulin resistance and obesity and effects of weight loss. FASEB J 13:2051-2060 